\title{
EARLY CHANGES IN THE GERMINAL EPITHELIUM OF RAT TESTES FOLLOWING EXPOSURE TO HEAT
}

\author{
A. K. GHOWDHURY AND E. STEINBERGER \\ Division of Endocrinology and Reproduction, Research Laboratories, \\ Albert Einstein Medical Center, Philadelphia, Pa. 19141
}

(Received 8th May 1969)

\begin{abstract}
Summary. Changes in the seminiferous epithelium of the rat testis occurring within $24 \mathrm{hr}$ after exposure to $43^{\circ} \mathrm{C}$ for $15 \mathrm{~min}$ were investigated using a quantitative technique. The earliest cytochemical or morphological changes were detected in the pachytene spermatocytes in stages IX to XII, the diakinetic and dividing spermatocytes in stages XIII and XIV and the young spermatids at stage I of spermatogenesis within $\mathrm{I} \mathrm{hr}$ after exposure. The spermatocytes showed changes in the cytoplasm and the spermatids, in the nucleus.

The number of abnormal spermatocytes increased progressively with time after the exposure and the damage was detectable in more stages at later time intervals. Within $4 \mathrm{hr}$ after exposure, the damage could also be detected in pachytene spermatocytes at stages VII and VIII, as well as through all the stages up to XIV. The pachytene, diakinetic and dividing spermatocytes from stages IX to XIV were essentially absent $24 \mathrm{hr}$ after the exposure, and those at stages VII and VIII showed a drastic reduction in count. Similarly, a progressive increase in the number of abnormal spermatids was noted, associated with an intensification of the morphological changes in the nucleus characterized by typical ring formation pyknosis and chromatolysis. The sequence of degenerative changes varied considerably between spermatids and spermatocytes-the former showing the earliest changes in the nucleus, and the latter in the cytoplasm.

These findings extend and confirm earlier observations on the specific susceptibility of testicular germinal cells to heat. The pachytene, diakinetic and dividing spermatocytes at stages IX to XIV and the young step-1 spermatids are most susceptible to heat under the described experimental conditions.
\end{abstract}

\section{INTRODUCTION}

The lack of clear understanding of the kinetics of spermatogenesis and of appropriate guide lines for identification of the various types of cells of the germinal epithelium limited early attempts at precise localization of cellular changes in the mammalian testisfollowing exposure to heat. Results based on qualitative histological studies of changes in testicular morphology led to contradictory conclusions (Fukui, 1923; Moore, 1924; Young, 1927; Asdell \& Salisbury, 
1941; Mori, 1951; Nelson, 1951), failing to resolve the problem of the heat susceptibility of specific types of cells of the germinal epithelium.

Steinberger \& Dixon (1959) have shown that a single 15-min exposure of the rat testis to $45^{\circ} \mathrm{C}$ produced extensive, non-specific damage to various types of germinal epithelium cells detectable $4 \mathrm{hr}$ after exposure, but a 15-min exposure to $43^{\circ} \mathrm{C}$ produced specific damage limited to the spermatocytes. Subsequently, Chowdhury \& Steinberger (1964), using quantitative methods for analysis of the germinal epithelium, confirmed this observation and identified precisely the heat-susceptible cells at various stages of the spermatogenic process. Once the heat-susceptible cells were known, it was of interest to examine how soon these cells exhibit morphological or cytochemical changes after heat exposure. The present study was, therefore, conducted to investigate the changes occurring in the seminiferous epithelium during the first $24 \mathrm{hr}$ after exposure to heat.

\section{MATERIALS AND METHODS}

Adult, hooded rats of Long-Evans strain bred in our laboratory were used. They were housed in conventional rat cages in an animal room maintained at approximately $26^{\circ} \mathrm{C}$. Rockland rat chow and water were supplied freely.

The animals were anaesthetized and the scrotum was exposed to $43^{\circ} \mathrm{C}$ for $15 \mathrm{~min}$. The detailed techniques of heat exposure have already been described (Steinberger \& Dixon, 1959). Groups of animals were killed immediately (the zero-hour group), and 1, 2, 4 and $24 \mathrm{hr}$ after exposure. Control groups of animals were anaesthetized but not exposed to heat. The testes were fixed in Bouin's fluid and processed, employing the conventional methods for obtaining paraffin sections cut at 3 to $4 \mu$. PAS-haematoxylin technique was used for staining the sections. The procedure for quantitative analysis of the seminiferous epithelium in testicular sections has already been described (Chowdhury \& Steinberger, 1964).

\section{RESULTS}

The testes of animals killed immediately after exposure, i.e. at zero-hour, appeared slightly congested but no histological abnormalities were observed.

TABLE 1

NUMBER OF SPERMATOGONIA PER TUBULAR GROSS-SECTION AT VARIOUS TIME INTERVALS AFTER EXPOSURE OF RAT TESTES TO $43^{\circ} \mathrm{G}$ FOR 15 MIN

\begin{tabular}{|c|c|c|c|c|c|c|c|}
\hline $\begin{array}{c}\text { Type of } \\
\text { spermatogonia }\end{array}$ & Stage & Control & $0 h r$ & $1 \mathrm{hr}$ & $2 h r$ & $4 h r$ & $24 h r$ \\
\hline $\begin{array}{c}\mathrm{A} \\
\mathrm{A} \\
\mathrm{A} \\
\mathrm{A} \\
\mathrm{A} \text { and I } \\
\mathrm{A} \text { and I } \\
\mathrm{A}, \mathrm{I} \text { and } \mathrm{B} \\
\mathrm{B}\end{array}$ & $\begin{array}{l}\text { VII } \\
\text { IX } \\
\text { XII } \\
\text { XIV } \\
\text { I } \\
\text { III } \\
\text { IV } \\
\text { V }\end{array}$ & $\begin{array}{r}1.2 \pm 0.7 * \\
2.9 \pm 0.9 \\
3.0 \pm 1.0 \\
3.9 \pm 1.0 \\
6.8 \pm 1.5 \\
8.5 \pm 2 \cdot 3 \\
14.7 \pm 3 \cdot 8 \\
25.5 \pm 4.2\end{array}$ & $\begin{array}{r}1 \cdot 0 \pm 0.9 \\
2 \cdot 8 \pm 1 \cdot 0 \\
2 \cdot 9 \pm 1 \cdot 2 \\
4 \cdot 1 \pm 1 \cdot 0 \\
6 \cdot 8 \pm 1 \cdot 2 \\
8 \cdot 5 \pm 2 \cdot 0 \\
15 \cdot 1 \pm 2 \cdot 8 \\
24 \cdot 1 \pm 2 \cdot 5\end{array}$ & $\begin{array}{r}1.2 \pm 0.9 \\
2 \cdot 0 \pm 0.9 \\
3 \cdot 1 \pm 1 \cdot 0 \\
3.9 \pm 1 \cdot 0 \\
6.8 \pm 1.5 \\
8.9 \pm 1 \cdot 8 \\
14.5 \pm 3.8 \\
23.0 \pm 3.1\end{array}$ & $\begin{array}{r}1.0 \pm 0.8 \\
2.5 \pm 0.5 \\
3.0 \pm 0.9 \\
3.9 \pm 0.9 \\
7.0 \pm 1.2 \\
9.1 \pm 1.1 \\
14.6 \pm 3.8 \\
23.8 \pm 3.1\end{array}$ & $\begin{array}{r}1.3 \pm 0.6 \\
2.9 \pm 0.9 \\
3 \cdot 1 \pm 0.9 \\
4.2 \pm 0.9 \\
6.9 \pm 1.5 \\
8.9 \pm 2 \cdot 1 \\
14.6 \pm 3.5 \\
23.5 \pm 4 \cdot 1\end{array}$ & $\begin{array}{r}1.2 \pm 0.9 \\
2 \cdot 8 \pm 0.9 \\
2.9 \pm 1.0 \\
4.0 \pm 1 \cdot 1 \\
6.5 \pm 1.2 \\
8.6 \pm 2.5 \\
14.7 \pm 3.5 \\
23.6 \pm 4 \cdot 2\end{array}$ \\
\hline
\end{tabular}

*Mean \pm S.E. 


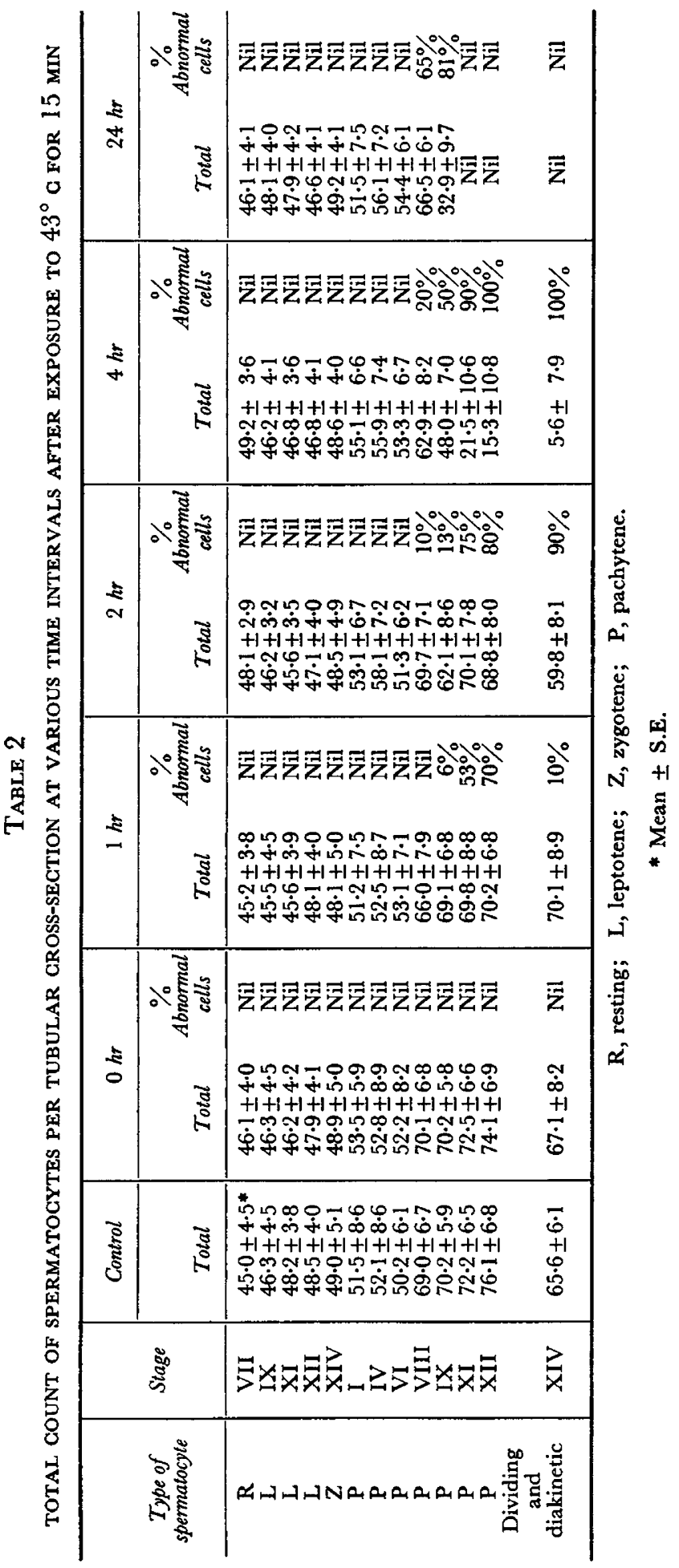


The frequency distribution of the different stages of spermatogenesis in all the experimental groups of animals was comparable to that of the control testes. The morphological characteristics and the counts of the spermatogonia remained unchanged (Table 1). Sertoli and Leydig cells were also morphologically normal at all time intervals after exposure.

\section{One hour after exposure}

Spermatids. Within $1 \mathrm{hr}$ after exposure, some step-1 spermatids developed definite morphological changes characterized by the irregular size and shape of the nucleus containing coarse chromatin granules. There was, however, no visible change in the cytoplasm (Pl. 1, Fig. 1). All other spermatids appeared normal.

Spermatocytes. The count of pachytene spermatocytes was comparable to those of the control, but some of these cells showed an abnormal cytochemical reaction characterized by a PAS-positive cytoplasm and intensely basophilic nuclei (Pl. 1, Fig. 2). The number of such abnormal cells increased progressively from stage IX $(6 \%)$ to stage XII $(70 \%)$ (Table 2$)$. The dividing spermatocytes at stage XIV were mostly normal, but a few of these cells also developed a faint PAS-positive cytoplasm (Pl. 1, Fig. 3).

\section{Two hours after exposure}

Spermatids. Within $2 \mathrm{hr}$ after exposure, a large number of step-1 spermatids in stage I developed nuclear changes similar to those already described. All other spermatids, however, appeared normal.

Spermatocytes. Some pachytene spermatocytes at stage VIII showed a positive PAS reaction in the cytoplasm. While the total count of these cells at stage VIII

\section{EXPLANATION OF PLATE 1}

Cross-sections of spermatic tubules. Roman numerals indicate the stages of spermatogenesis; A, B and $C$ indicate time after heat exposure. All sections stained by PAS technique. $\times 960$.

A. 1 hr after exposure

Fic. 1. Note nuclear changes in the step-1 spermatids. The nuclear outline and size are irregular; the chromatin granules are unusually coarse.

Fig. 2. All cells appear normal except the pachytene spermatocyte marked with arrow. Its nucleus is pyknotic, intensely basophilic, and its cytoplasm shows a PAS-positive reaction indicated by the haze around the nucleus.

Frc. 3. The dividing spermatocytes show abnormal PAS reaction in the cytoplasm (arrow).

\section{B. 4 hr after exposure}

Fig. 4. The step-1 spermatids show typical ring formation (arrow).

Fig. 5. Most pachytene spermatocytes are degenerating and show pyknotic nuclei and PAS-positive cytoplasm. The pachytene spermatocyte marked with arrow shows normal nuclear morphology but intense PAS reaction in the cytoplasm.

FIG. 6. All the dividing spermatocytes are pyknotic and show a PAS-positive cytoplasm. C. 24 hr after exposure

Fig. 7. Note absence of step-1 spermatids, except for 'ghosts' (arrow).

Fic. 8. The pachytene spermatocytes show pyknosis and degeneration.

Fig. 9. The dividing spermatocytes are completely absent except for 'ghost cells' (arrow); other cells are normal. 
PIATE 1

IX

XIV

201

A 1968683

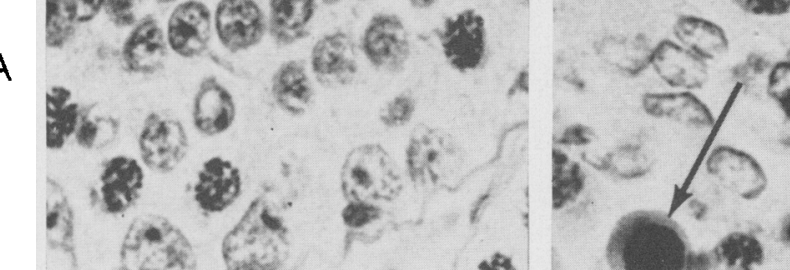

(8) is (1) 0." f.

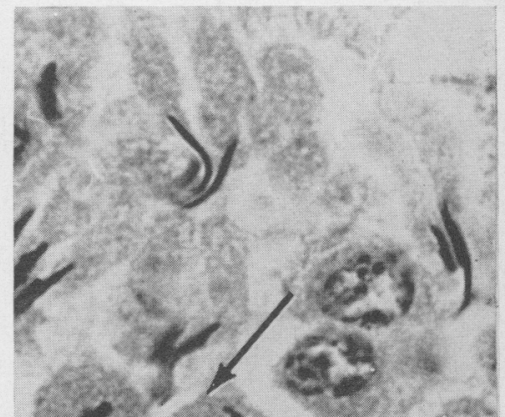

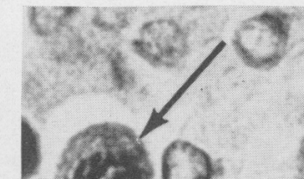
$=100$
$2-100$ $10^{2}$ o o 0

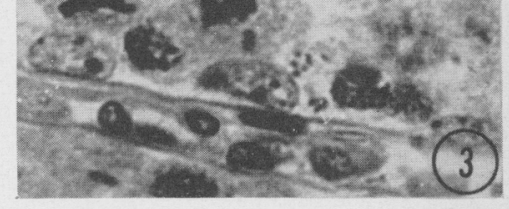

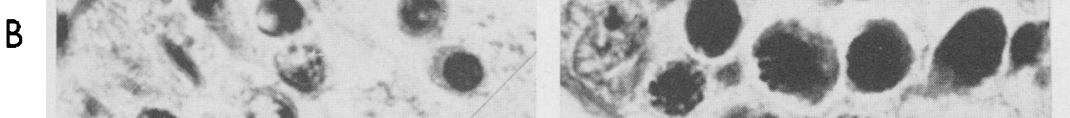

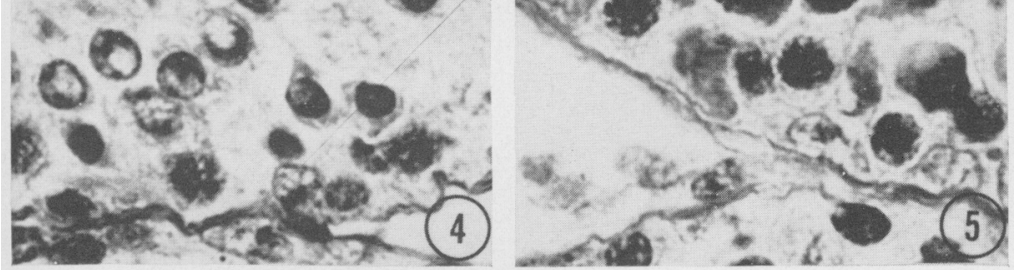<smiles>C1C[C@H]2C[C@H]1C2</smiles>

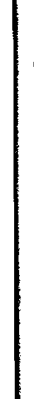
(2) $\left.\sin ^{1}\right|^{4}$

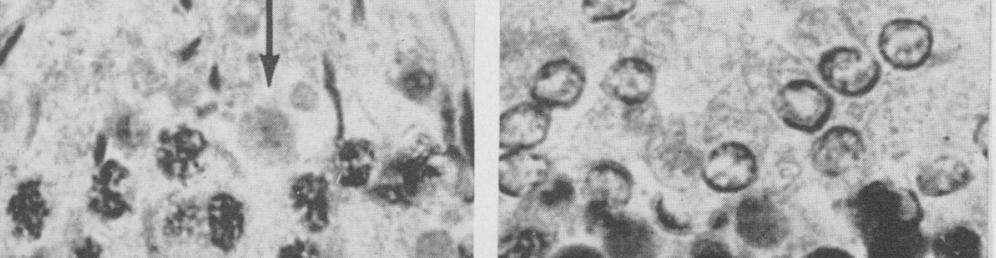
a. +2.3 .9$

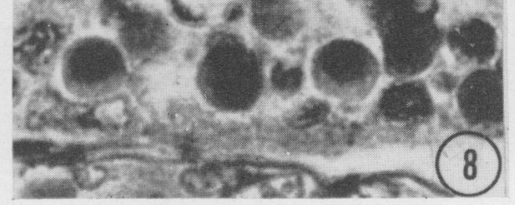

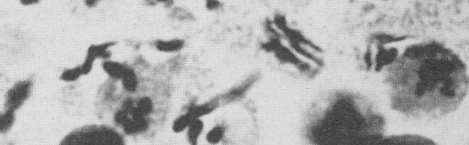

$24 x^{2}+x^{2}$

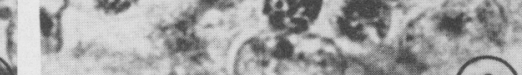

$$
=\ldots
$$

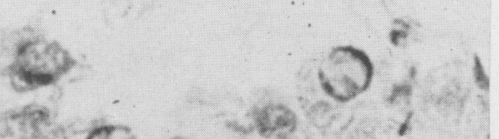

$\lim _{(12)}\left(\frac{1}{1}\right)^{2}$ - $\left.1 \int^{2}\right|^{\prime}=$ 2. 4 a 1 क S. 0.60 .5

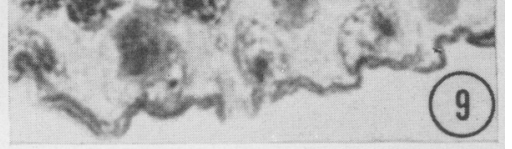


remained normal, about $10 \%$ showed the abnormal staining reaction. The number of abnormal pachytene spermatocytes increased progressively from stage IX to XII, the percentage of abnormal cells being $13 \%, 75 \%$ and $80 \%$, respectively. Pyknotic nuclei appeared in a large number of cells, showing a strongly positive PAS reaction in the cytoplasm. In some cells, the chromatin network was broken and chromatolysis and cytolysis were apparent. Fragmentation of the nuclear material was also seen in some cases. The number of pachytene spermatocytes at stages IX to XII showed a tendency to decline, although it is not very apparent from the mean value shown in Table 2. A considerable number of tubules showed only few pachytene spermatocytes and a number of 'ghost' cells staining faintly with PAS. The dividing and diakinetic spermatocytes at stage XIV showed marked degenerative changes, about $90 \%$ of the cells staining strongly with PAS and showing bizarre meiotic figures, pyknosis and chromatolysis.

\section{Four hours after exposure}

Spermatids. In step-1 spermatids, the nuclei were smaller than normal, the chromatin material aggregated thickly along the nuclear border, creating the 'ring' appearance in the nucleus. The chromatin appeared to be invariably denser at the pole near the idiosome (Pl. 1, Fig. 4). A few tubules at stage II of spermatogenesis showed similar changes in the step-2 spermatids.

Spermatocytes. At this time interval, pachytene spermatocytes from stages IX to XIV showed severe damage (Pl. 1, Figs. 5 and 6). A small number (20\%) of pachytene spermatocytes in stage VIII were also affected. The typical nuclear changes, pyknosis, chromatolysis, etc., could be seen associated with a PAS-positive cytoplasm. The count of pachytene spermatocytes also showed a decline. The pachytene spermatocytes at stages IX to XII showed extensive damage, 50 to $100 \%$ of the cells being abnormal in different tubular crosssections. The nucleus of the affected cells showed either pyknotic changes or chromatolysis (Pl. 1, Fig. 5). In some cells, chromatolysis was characterized by fragmentation of the chromatin material. In others, the pyknotic nuclei gradually lost their compactness and nuclear material diffused into the cytoplasm, probably due to damage to the nuclear membrane. The cytoplasmic PAS reaction decreased in intensity with advanced nuclear changes and ultimately, after complete chromatolysis, the cytoplasmic remnant remained detectable only as a faintly PAS-positive body designated a 'ghost cell'. A large number of degenerated cells were apparently removed by this time, as the count of pachytene spermatocytes at stage XII was about one-fifth of normal. Those present were invariably in different stages of degeneration. In stages VIII and IX, a larger number of pachytene spermatocytes was found to be abnormal in appearance compared with the $2-\mathrm{hr}$ group. In stage XIV, only a few dividing spermatocytes were present, but these cells were invariably abnormal, showing an intense PAS reaction of the cytoplasm along with nuclear pyknosis, chromatolysis and bizarre meiotic figures (Pl. 1, Fig. 6).

\section{Twenty-four hours after exposure}

Spermatids. The young spermatids were absent in stage I and in a few tubules 
at stage II (Pl. 1, Fig. 7). Beyond step 2 of spermiogenesis, all spermatids were normal in appearance.

Spermatocytes. By this time, pachytene spermatocytes at stage VII were affected. The cells showed the typical cytoplasmic and nuclear changes already described. Some tubules in stage VII contained very few pachytene spermatocytes. In stage VIII, the number of abnormal spermatocytes increased to about $65 \%$, although the total number of cells was not decreased. In a few tubules, however, the pachytene spermatocytes were completely absent. In stage IX, the total number declined to less than half the control value and about $81 \%$ of these cells were degenerating (Pl. 1, Fig. 8). In stages XII and XIII, the pachytene spermatocytes were almost completely absent. In stage XIV, the dividing spermatocytes also disappeared, leaving cellular débris and ghost cells (Pl. 1, Fig. 9).

\section{DISCUSSION}

It has been clearly demonstrated that mammalian spermatogenesis proceeds through well-defined stages, forming the so-called cycle of the seminiferous epithelium (Leblond \& Clermont, 1952). It has also been shown that by using the concept of spermatogenic cycle and its timing, it is possible to locate precisely the specific types of germinal cells affected by adverse agents, such as heat (Steinberger, 1962; Chowdhury \& Steinberger, 1964). The pattern of degeneration in different stages of spermatogenesis can be predicted if the time interval between exposure and autopsy is known, though the rate of degeneration of the susceptible cells varies considerably (Chowdhury \& Steinberger, 1964). On the basis of results from these experiments, the susceptible cells can be divided into three categories: (1) Cells which degenerate and disappear from the seminiferous tubules within 2 days after exposure (spermatids at step 1 of spermiogenesis; pachytene spermatocytes in stages VII to XII ; the diakinetic and dividing spermatocytes in stages XIII and XIV of spermatogenesis); (2) Cells which are apparently capable of some differentiation into more advanced stages before degeneration and disappearance (zygotene spermatocytes in stage XIV and pachytene spermatocytes in stages I to IV); (3) Cells which are capable of considerable development and differentiation, including division and formation of spermatids. These spermatids, however, disappear within 8 to 26 days after exposure (leptotene spermatocytes in stages IX to XII).

The present experiments were designed to study changes occurring in the first category of heat-susceptible cells during the first $24 \mathrm{hr}$ after exposure to heat. Since the duration of stage XII is approximately $32 \mathrm{hr}$, and since the pachytene spermatocytes were absent in stages XII and XIII $24 \mathrm{hr}$ after the exposure, these cells must have degenerated, failing to differentiate to the diakinetic phase of stage XIII. Similarly, stage I of spermatogenesis requires about $34 \mathrm{hr}$, and no step-1 spermatids were found within $24 \mathrm{hr}$ after exposure, indicating that these cells must have degenerated rapidly. On the other hand, pachytene spermatocytes in stage VII, which required about $63 \mathrm{hr}$ for completion, were unaltered $4 \mathrm{hr}$ after the exposure. A large number of them, however, developed typical degenerative changes within $24 \mathrm{hr}$ and many tubules in stage VII were devoid of pachytene spermatocytes after $24 \mathrm{hr}$. Thus, it appeared that 
pachytene spermatocytes in stage VII of spermatogenesis varied in the rate of degeneration and most probably the pachytene spermatocytes in the latter part of stage VII are more susceptible to heat than those in the early parts of this stage. The pachytene spermatocytes in stages IX to XII and the diakinetic and dividing spermatocytes in stages XIII and XIV are perhaps the most susceptible cells in this group, since they disappeared within a few hours after exposure, failing to undergo any further maturation. Although, $4 \mathrm{hr}$ after the exposure a majority of these cells underwent degeneration, some normallydividing spermatocytes were unexpectedly found in stage XIV producing apparently normal spermatids even $2 \mathrm{hr}$ after exposure. We do not have an explanation for this irregularity.

These results show that the most heat-sensitive cells are the pachytene, diakinetic and dividing spermatocytes in stages IX to XIV, and they actually show severe degenerative changes within 1 to $4 \mathrm{hr}$ after the exposure. The pachytene spermatocytes in stages VII and VIII also degenerate, but at a much slower rate--some of them remain apparently intact up to $24 \mathrm{hr}$ after exposure and may even pass to the next stage of spermatogenesis, but ultimately they also degenerate (Chowdhury \& Steinberger, 1964). Examination of spermatocytes in other stages of spermatogenesis showed no detectable abnormalities during the first $24 \mathrm{hr}$ after exposure, except for some morphological nuclear changes in some pachytene spermatocytes found in stage I of spermatogenesis. It is known that the pachytene spermatocytes at this stage of spermatogenesis are in a state of transition from zygotene to typical pachytene. Possibly, the heat exposure adversely affects the nuclear reorganization required for this change. Although during the first $24 \mathrm{hr}$ there were signs of nuclear changes in these cells they were able to progress up to stage III of spermatogenesis. From previous studies, however, it is known that they did not proceed further with development but degenerated (Chowdhury \& Steinberger, 1964).

The degenerative changes in spermatocytes followed a different pattern from that observed in spermatids. In spermatocytes, the earliest detectable change was an abnormal cytochemical reaction in the cytoplasm and nuclear changes were not apparent until the cytoplasmic changes became intense. In spermatids, on the other hand, the initial changes occurred in the nucleus, where the chromatin granules appeared to accumulate around the nuclear membrane. The nuclear membrane subsequently thickened and a vacuole formed in the centre of the nucleus, the chromatin material having accumulated more at one pole of the nucleus than at the other. This pole was invariably near the Golgi zone or area of further development of the acrosomic granule. With time, the entire nucleus shrank, forming a compact mass around the vacuole. In this fashion, the classic 'ring formation' of the spermatid nuclei took place.

Various investigators have studied the degeneration of the germinal epithelium of mammalian testes exposed to heat. It has been proposed by some that heat affects the mechanism of cell division and that the testicular degeneration is due to maturation depletion (Guensel, 1949). The present work shows that in addition to cells in the final process of division, the primary spermatocytes at various steps of the meiotic process are also susceptible to heat, and even if they do not show immediate degenerative changes after the exposure, they 
do degenerate after they have attained a certain step in their development. The absence of morphological abnormality immediately or shortly after exposure indicates that heat may affect some metabolic activity in the susceptible cell, which does not cause morphologically visible damage until the cell achieves a certain stage in its development. The appearance of PAS-positive material in the cytoplasm in degenerating primary spermatocytes may be an expression of such metabolic damage. While it is quite clear that the meiotic process is highly susceptible to heat, neither the experiments reported here nor the data reported previously succeed in clarifying the resistance to heat of spermatocytes at certain stages of the meiotic process, mainly of pachytene spermatocytes in stages $\mathrm{V}$ and VI of spermatogenesis.

The study also clearly establishes the fact that the young spermatid-a cell which is not undergoing division, nor preparing to do so--is also highly susceptible to heat. The mechanism here, however, appears to be of an entirely different nature, as suggested by the fact that the morphological changes are entirely different in the affected spermatid from those found in the affected spermatocyte.

The studies reported here provide information concerning the cytological localization of heat damage in the germinal epithelium. They show that at certain heat levels, specific types of cells only are affected, but throw no light on the biochemical mechanisms responsible for the change and ultimate death of the affected cells.

\section{ACKNOWLEDGMENTS}

This work was performed under the auspices of a Postdoctoral Fellowship awarded to A. K. G. by the Population Council and further supported by Grant AM 05449 from the National Institutes of Health, U.S. Public Health Service.

\section{REFERENCES}

Asdell, S. A. \& Salisbury, G. W. (1941) The rate at which spermatogenesis occurs in the rabbit. Anat. Rec. 80, 145.

Ghowdhurx, A. K. \& Steingerger, E. (1964) A quantitative study of the effect of heat on the germinal epithelium of the rat testis. Am. $\mathcal{7}$. Anat. 115, 509.

Fukur, N. (1923) On the action of heat rays upon the testicles. An histologic, hygienic and endocrine study. Acta Sch. med. Univ. Kioto, 6, 225.

GuENSEL, E. (1949) Über die warmeempfindlichkeit dis keimepithels im Rattenhoden. Strahlentherapie, 80, 299.

Leblond, C. P. \& Glermont, Y. (1952) Definition of stages of the cycle of the seminiferous epithelium in the rat. Ann. N.Y. Acad. Sci. 55, 548.

Moore, C. R. (1924) Properties of gonads as controller of somatic and physical characteristics. VI. Testicular reaction in experimental cryptorchidism. Am. F. Anat. 34, 269.

MoRI, A. (1951) Studies on abnormal spermatozoa. I. Appearance of abnormal spermatozoa in the experimental cryptorchidism of albino rats. Tohoku F. agric. Res. 1, 15.

Nelson, W. O. (1951). Mammalian spermatogenesis: effect of experimental cryptorchidism. Recent Prog. Horm. Res. 6, 29.

Steinberger, E. (1962) A quantitative study of the effect of an alkylating agent (triethylenemelamine) on the seminiferous epithelium of rats. $\mathcal{F}$. Reprod. Fert. 3, 250.

Steinberger, E. \& Dixon, W. J. (1959) Some observations on the effect of heat on the testicular germinal epithelium. Fert. Steril. 10, 578.

YounG, W. C. (1927) The influence of high temperature on the guinea pig testis. F. exp. Zool. 49, 459. 